\title{
COMMENT
}

\section{Goblet cells promote tolerance induction in the conjunctiva}

\author{
Stephen C. Pflugfelder $\mathbb{D}^{1}$ and Cintia S. de Paiva ${ }^{1}$ \\ Mucosal Immunology (2020) 13:717-718; https://doi.org/10.1038/s41385-020-0319-1
}

We commend Kulkarni et al. for their excellent study entitled published in the 2020, volume 13, issue 2 of Mucosal Immunology (ref. ${ }^{1}$ ) showing the importance of goblet cell-associated passages (GAPs) in inducing immune tolerance to antigens in the intestinal lumen. ${ }^{1}$ Our previously reported study found goblet cells also support the induction of conjunctival immune tolerance. ${ }^{2}$ Immune tolerance on the ocular surface is essential for maintaining corneal clarity and quality vision. It is recognized that topical antigen application to the ocular surface induces potent immune tolerance, through passive and active mechanisms. ${ }^{3,4}$

Goblet cells are specialized, secretory cells that produce mucins and other supportive and protective factors. Goblet cells are found in many wet mucosal tissues, including the nose, large airways, intestine, and conjunctiva. Among these, the distal colon and conjunctiva are the most goblet cell dense tissues. ${ }^{5}$ The predominant mucin produced by conjunctival goblet cells is Muc5ac, in contrast to the gut where Muc2 expression is higher than Muc5ac. ${ }^{5}$ Conjunctival goblet cells have also been found to produce immunomodulatory factors, such as TGF- $\beta 2$ and retinoic acid (RA).,

Conjunctival goblet cells are essential for the maintenance of ocular surface health. Aqueous deficient dry eye and inflammatory diseases, such as Stevens-Johnson syndrome, graft-versus-hostdisease, and aging are accompanied by significant conjunctival goblet cell loss. ${ }^{8-10}$ Goblet cell density not only correlates with the severity of the ocular surface disease, but the loss of goblet cells appears to perpetuate a cycle of chronic ocular surface inflammation. ${ }^{11-13}$ These inflammatory diseases carry a significant burden to the individual and society.

Following the initial report of GAPs by Knoop et al., ${ }^{14}$ we found that conjunctival goblet cells also function as antigen passages. ${ }^{4}$ Smaller fluorescent molecules $(\leq 10 \mathrm{kDa})$ showed greater passage through conjunctival gaps than larger ones (70 kDa), and topically applied 2.3 kDa ovalbumin (OVA) antigen was directed by GAPs to subepithelial CD11 $\mathrm{b}^{+}$phagocytic antigen-presenting cells (APCs). Furthermore, OVA applied to the conjunctiva of Spdef $^{-1}$ mice that lack goblet cells had greater retention of antigen in the epithelium and significantly fewer $\mathrm{OVA}^{+} \mathrm{CD} 11 \mathrm{~b}^{+}$cells in the conjunctiva. We compared the ability to induce conjunctival immune tolerance in wild-type and Spdef ${ }^{-1-}$ strains. An increased number of conjunctival APCs and IL- $12^{+} \mathrm{CD} 11 \mathrm{~b}^{+}$cells was found in the Spdef $^{-\prime-}$ conjunctiva. $^{2}$ Application of OVA eyedrops (as a surrogate antigen) to the ocular surface induced conjunctival immune tolerance in the wild-type strain as expected, but this was not observed in Spdef $^{-1-}$ mice ${ }^{2}$ There was a greater generation of IFN- $\gamma$ producing CD4+ cells and a decrease in $\mathrm{CD}^{+}{ }^{+} \mathrm{Foxp}^{+}$cells in Spdef $^{-\prime-2}$. Our study delineates the importance of the goblet cells in maintaining mucosal tolerance, as was the case in the study performed by Kulkarni et al. ${ }^{1}$ It also indicates that goblet cells in different mucosal sites may have a similar tolerance inducing function.

Kulkarni found that RA may be important in tolerance induction. ${ }^{1}$ Similar to the intestine, the ocular surface is retinoid rich. Retinol secreted by the lacrimal gland into tears is metabolized by aldehyde dehydrogenase enzymes in the conjunctival goblet cells to RA that conditions APCs. We also reported that goblet cell-conditioned media had similar activity as RA in decreasing IL-12 and IFN- $\gamma$ production in stimulated bone marrow derived myeloid cells in vitro.?

Taken together, these findings support the concept that goblet cells serve as antigen passages that function in immunetolerance induction in goblet cell containing mucosa, like the intestine and conjunctiva, and highlight the importance of goblet cell preservation as a therapeutic strategy in mucosal inflammatory disease.

\section{ACKNOWLEDGEMENTS}

This work was supported by NIH Grant EY11915 (SCP), NIH Core Grants-EY002520 \& EY020799, Pathology Cell Core P30CA125123, Biology of Inflammation Center Baylor College of Medicine, an unrestricted grant from Research to Prevent Blindness, New York, NY (SCP), the Oshman Foundation, Houston, TX (SCP), the William Stamps Farish Fund, Houston, TX (SCP), Hamill Foundation, Houston, TX (SCP), Sid W. Richardson Foundation, Ft Worth, TX (SCP).

\section{AUTHOR CONTRIBUTIONS}

Both authors conceived this letter and participated equally in writing.

\section{ADDITIONAL INFORMATION}

Competing interests: The authors declare no competing interests.

Publisher's note Springer Nature remains neutral with regard to jurisdictional claims in published maps and institutional affiliations.

\section{REFERENCES}

1. Kulkarni, D. H. et al. Goblet cell associated antigen passages support the induction and maintenance of oral tolerance. Mucosal Immunol. 13, 271-282 (2020).

2. Ko, B. Y., Xiao, Y., Barbosa, F. L., de Paiva, C. S. \& Pflugfelder, S. C. Goblet cell loss abrogates ocular surface immune tolerance. JCl insight 3, 98222 (2018).

3. Egan, R. M. et al. In vivo behavior of peptide-specific $T$ cells during mucosal tolerance induction: antigen introduced through the mucosa of the conjunctiva elicits prolonged antigen-specific T cell priming followed by anergy. J. Immunol. 164, 4543-4550 (2000).

4. Barbosa, F. L. et al. Goblet cells contribute to ocular surface immune toleranceimplications for dry eye disease. Int J. Mol. Sci. 18, 1-13. (2017).

5. McCauley, H. A. \& Guasch, G. Three cheers for the goblet cell: maintaining homeostasis in mucosal epithelia. Trends Mol. Med 21, 492-503 (2015).

${ }^{1}$ Department of Ophthalmology, Baylor College of Medicine, Houston, TX, USA

Correspondence: Stephen C. Pflugfelder (stevenp@bcm.edu)

Received: 26 May 2020 Accepted: 21 June 2020

Published online: 2 July 2020 
Goblet cells promote tolerance induction in the conjunctiva SC Pflugfelder and CS de Paiva

718

6. Contreras-Ruiz, L. \& Masli, S. Immunomodulatory cross-talk between conjunctival goblet cells and dendritic cells. PLoS One 10, e0120284 (2015).

7. Xiao, Y. et al. Goblet cell-produced retinoic acid suppresses CD86 expression and IL-12 production in bone marrow-derived cells. Int Immunol. 30, 457-470 (2018).

8. Bron, A. J. et al. TFOS DEWS II pathophysiology report. Ocular Surf. 15, 438-510 (2017).

9. Whaley, K. et al. Sjogren's syndrome and autoimmunity in a geriatric population. Age Ageing 1, 197-206 (1972).

10. Ralph, R. A. Conjunctival goblet cell density in normal subjects and in dry eye syndromes. Invest Ophthalmol. 14, 299-302 (1975).
11. Pflugfelder, S. C. et al. Correlation of goblet cell density and mucosal epithelial membrane mucin expression with rose bengal staining in patients with ocular irritation. Ophthalmology 104, 223-235 (1997).

12. Pflugfelder, S. C. et al. Aqueous tear deficiency increases conjunctival interferon- $\gamma$ (IFN- $\gamma$ ) expression and goblet cell loss. Invest Ophthalmol. Vis. Sci. 56, 7545-7550 (2015).

13. Pflugfelder, S. C., et al. Severity of Sjogren's syndrome keratoconjunctivitis sicca increases with increased percentage of conjunctival antigen-presenting cells. Int $J$ Mol Sci. 19, 2760-2769 (2018).

14. McDole, J. R. et al. Goblet cells deliver luminal antigen to $\mathrm{CD} 103+$ dendritic cells in the small intestine. Nature 483, 345-349 (2012). 